\title{
Sakurai's Object: Spectroscopic Monitoring and Nebula Observations
}

\author{
D.L. Pollacco ${ }^{1}$, N.A. Walton ${ }^{1}$, H.G. Schwarz ${ }^{2}$ and S.A. Bell ${ }^{3}$ \\ ${ }^{1}$ Isaac Newton Group, La Palma; ${ }^{2}$ Nordic Optical Telescope, La Palma; \\ ${ }^{3}$ Royal Greenwich Observatory, Cambridge
}

Sakurai's object is thought to be undergoing a final thermal pulse or shell flash. It is the first example of this type of object to be studied using modern instrumentation and will be useful and unique for the time being for placing theoretical models of these transient events on a firm observational footing. The only other definite candidate is V605 Aql - now the WC central star of the planetary nebula Abell 58 - which is thought to have undergone a final thermal pulse around 1918.

It is against this background that we have started intensive observations of the "novalike object in Sagittarius" (Nakano, 1996) also known as Sakurai's object. In early 1995, it lay below 16th magnitude and rose to 11 th magnitude during 1995-96. Since February 1996, we have obtained spectra every few weeks in an attempt to follow the spectroscopic evolution of this object. The growing strength of the $\mathrm{H} \alpha$ line indicates that the star has been rapidly warming from approximately $5000 \mathrm{~K}$ to $8000 \mathrm{~K}$ in an interval of 5 months. We will continue to monitor this object through the coming months (and hopefully years).

Very recently, we have also obtained 2-dimensional spectroscopy and deep imaging of the nebula surrounding Sakurai's object with the 4.2-m William Herschel Telescope. Initial impressions suggest an expansion rate of some $28 \mathrm{kms}^{-1}$ although we have yet to reduce and analyse these data properly. Observations also indicate that the nebula has faded considerably since monitoring began in February indicating that recombination has probably begun. Spectra obtained earlier this year suggest that the nebula exhibits relatively normal abundances. An analysis of the first season of data will be submitted for publication in Monthly Notices of the Royal Astronomical Society at the end of this year.

REFERENCES

Nakano, S., 1996, IAUC 6322. 